\title{
A technical framework for human-like motion generation with autonomous anthropomorphic redundant manipulators
}

\author{
Giuseppe Averta $^{1,2,3}$, Danilo Caporale ${ }^{1}$, Cosimo Della Santina ${ }^{4}$, Antonio Bicchi ${ }^{1,2,3}$, and Matteo Bianchi ${ }^{1,3}$
}

\begin{abstract}
The need for users' safety and technology acceptability has incredibly increased with the deployment of co-bots physically interacting with humans in industrial settings, and for people assistance. A well-studied approach to meet these requirements is to ensure human-like robot motions. Classic solutions for anthropomorphic movement generation usually rely on optimization procedures, which build upon hypotheses devised from neuroscientific literature, or capitalize on learning methods. However, these approaches come with limitations, e.g. limited motion variability or the need for high dimensional datasets. In this work, we present a technique to directly embed human upper limb principal motion modes computed through functional analysis in the robot trajectory optimization. We report on the implementation with manipulators with redundant anthropomorphic kinematic architectures - although dissimilar with respect to the human model used for functional mode extraction - via Cartesian impedance control. In our experiments, we show how human trajectories mapped onto a robotic manipulator still exhibit the main characteristics of human-likeness, e.g. low jerk values. We discuss the results with respect to the state of the art, and their implications for advanced human-robot interaction in industrial co-botics and for human assistance.
\end{abstract}

\section{INTRODUCTION}

Recent advancements in robotic design and control have opened promising opportunities in the field of Human-Robot Interaction (HRI), enabling for novel forms of assistance and collaborations e.g. in industrial co-botics or for personal assistance [1]. This perspective change has come with new challenges, e.g. the need for generating meaningful robot actions, simultaneously ensuring human ergonomics and safety, production quality, and users' acceptance.

One of the most studied solutions in literature to successfully tackle the aforementioned points is to guarantee the Human-Likeness (HL) of robot motions [2], [3], [4], [5], [6], [7]. This has been commonly pursued moving from the analysis of a large neuroscience and kinesiology literature, with the goal of devising suitable cost functions, whose optimization can likely ensure human-like characteristics in the planned motion [8]. In the state of the art, different descriptors for anthropomorphic movements have been proposed; for example, the presence of a single peak in the velocity profile

* This project has received funding from the European Union's Horizon 2020 research and innovation programme under grant agreement No. 688857 (SoftPro), 810346 (Natural BionicS) and 871237 (Sophia), and and by the Italian Ministry of Education and Research (MIUR) in the framework of the CrossLab project (Departments of Excellence).

${ }^{1}$ Centro di Ricerca "Enrico Piaggio", Universita d di Pisa, Largo Lucio Lazzarino 1, 56126 Pisa, Italy giuseppe.averta@ing.unipi.it

2 Soft Robotics for Human Cooperation and Rehabilitation, Fondazione Istituto Italiano di Tecnologia, via Morego, 30, 16163 Genova, Italy

3 Dipartimento di Ingegneria dell'Informazione, Universita‘ di Pisa, Largo Lucio Lazzarino 1, 56126 Pisa, Italy

4 MIT Computer Science Artificial Intelligence Lab, Massachusetts Institute of Technology, Cambridge, MA, USA

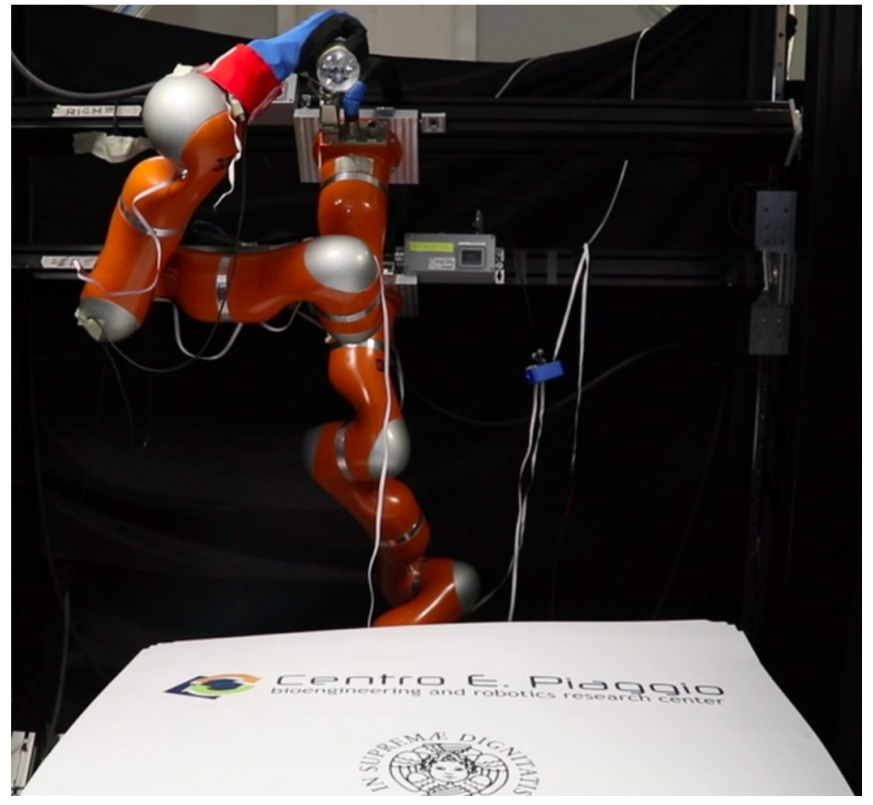

Fig. 1. A Kuka LWR+ 7 DoFs robot "drinking" water from a bottle.

was identified as a recurrent characterizing feature in point-topoint human arm motions [9]. In [10], Flash et al. theorized that a good approximation of human motion can be achieved through jerk minimization, and different robotic planning algorithms have been devised accordingly, see e.g. [11]. In [12], the author extended the minimum jerk and the minimum torque-change methods to account also for self- or externalimposed endpoint constraints, i.e. in terms of orientation and velocity. Another approach, presented in [13], proposed to use an arbitrary basis of discrete sine transformations, and expansion- function series, to generate complex movements via combination of basis elements.

Observations of human movements have been also used with learning methods, e.g. to train Neural Networks [14], [15], [16], [17], or to compute a set of parameters for non-linear dynamical systems [18], with specific focus on locomotion and whole-body characters animation[19], [16].

However, all the previously discussed methods come with some disadvantages. Model-based approaches, e.g. minimum jerk minimization, rely on the optimization of cost functions that are only a rough approximations of the real phenomenon [20]; this may limit the variability of the planned movement. On the other side, the efficiency and reliability of learning techniques is highly dependent on the quality and the dimensionality of the dataset used to train the network(s). 
To overcome these limitations for the generation of anthropomorphic robotic manipulative actions, we propose to directly embed human principal motion modes in the optimization algorithm. This principal modes are computed by applying functional analysis (functional Principal Component Analysis, fPCA) at the joint level, on a set of human recorded motions [21]. This approach comes with several advantages, since no hypothesis (or large dataset) is required to ensure HL, which is intrinsically guaranteed. Furthermore, this methodology comes with a significant perspective shift: from the search for optimal paths to the identification of a reduced number of scalars weighting the functional components. This could enable to rapidly achieve a solution for the planner, which is by definition human-like.

However, this method, theoretically introduced in [22], faces important translational changes, when it comes to be applied to robotic anthropomorphic manipulators, whose kinematic structure is dissimilar with respect to the model used for extracting human principal modes.

In this paper, we tackle the problem of mapping these modes on a generic anthropomorphic robotic manipulator. This problem is not trivial, since we cannot rely on jointbased one-by-one mapping and, at the same time, we must guarantee that the mapped trajectories still maintain the human-likeliness. Classic mapping algorithms implement optimization-based methods to maximise the similarity between the human and robot kinematic chain motions, see e.g. [23] and [24]. These methods can map human movements onto robotic structures, but these can be hardly used on-line. Here we propose a control-based approach that allows to implement onto a redundant robotic arm with a given kinematic architecture the trajectories obtained using the functional approach reported in [22], overcoming the limitations of the optimization methods described above. This approach relies on two Cartesian impedance controllers, which also allow to manage the redundancy problem: the first controller ensures that the robot end effector trajectories follow the ones of the human end-effector, while the second controller implemented at the elbow level projects in the null-space of the endeffector Jacobian. This implementation enables to keep the robot elbow as close as possible to the reference-human elbow trajectory - which is crucial for increasing HL [25], [26]- with no influence on the trajectory tracking at the endeffector level - controlled by the first control law. We report the details of this approach and show the implementation on an anthropomorphic robotic manipulator KUKA LWR+ platform, whose kinematic architecture is different from the kinematic model used to extract human functional modes. We discuss the experimental outcomes in terms of HL as well as the implications for collaborative robotics.

\section{BACKGROUND}

\section{A. Problem Definition}

In [21] and [22] we presented a mathematical model to characterize human upper limb movements, introducing a new method to generate human-inspired motions.

In this section we will briefly recall the main concepts of this idea. Our starting point is the coding of the main modes of variation of upper limb motions through functional Principal Component Analysis, namely (fPCA). From a dataset of

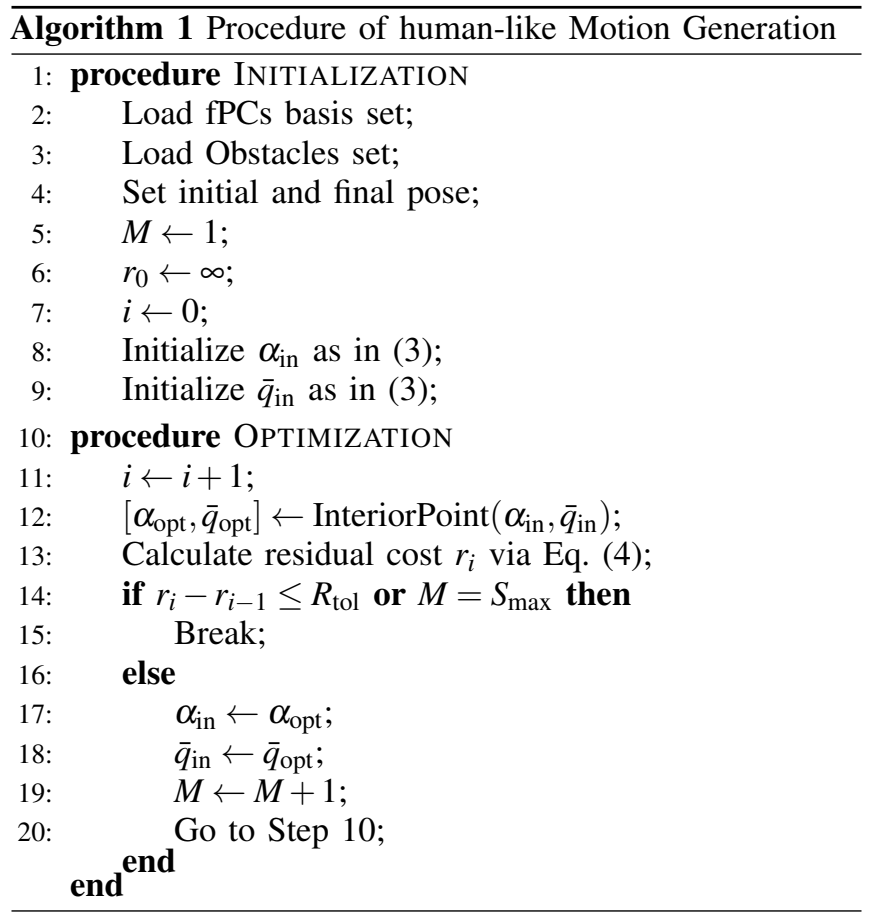

recorded joint movements, we extract a basis of functions whose combination can reconstruct any movement at the joint level. One of the properties of this basis is that the elements are associated with a specific cardinality, i.e. the first is the function that accounts for the higher percentage of motion variability, and so far. The larger is the number of the basis elements recruited, the higher is the accuracy of the reconstructed motion.

Leveraging on this, it is possible to devise a motiongeneration algorithm. More specifically, instead of searching for a solution in a generic infinite dimensional space $C_{n}^{1}\left[0, t_{\text {fin }}\right)$, where $n$ is the number of degrees of freedom of the considered model (in our case $n=7$ ), we define the possible arm movement as the finite dimensional family of functions

$$
q(t)=\bar{q}+S_{0}\left(\frac{t}{t_{\text {fin }}}\right)+\sum_{i=1}^{M} \alpha_{i} \circ S_{i}\left(\frac{t}{t_{\text {fin }}}\right),
$$

where $t$ is the time coordinate, $t_{\text {fin }}$ is the final time frame, $\bar{q} \in \mathbb{R}^{n}$ is the average posture of the recorded dataset, $S_{i}(t) \in$ $\mathbb{R}^{\mathrm{n}}$ is the $\mathrm{i}$-th element of the basis set, $\alpha_{i} \in \mathbb{R}^{\mathrm{k}}$ is a vector of weights, $S_{0}: \mathbb{R} \rightarrow \mathbb{R}^{7}$ is the zero-order synergy, calculated as the average movement, $n$ is the number of joints in the kinematic chain and $M$ the number of enrolled basis elements. The operator $\circ$ defines the Hadamard product, i.e. an elementwise product. Any movement can be generated by solving the following general optimization problem:

$$
\begin{array}{ll}
\min _{\bar{q}, \alpha_{1}, \ldots, \alpha_{M}} & J_{\text {task }}(q) \\
\text { subject to } & q(t)=\bar{q}+S_{0}\left(\frac{t}{t_{\text {fin }}}\right)+\sum_{i=1}^{M} \alpha_{i} \circ S_{i}\left(\frac{t}{t_{\text {fin }}}\right)
\end{array}
$$

where $J_{\text {task }}(q)$ is a task-dependent cost function. This approach reduces the search space - since it targets the 
computation of a reduced number of scalar weights for the functional components - and intrinsically ensures HL.

\section{B. Point-to-Point and constrained motions}

We demonstrated in [22] that the generation of free pointto-point movements results in a closed form solution. In particular, we can generate a zero-error point-to-point free motion with only one synergy using the following parameters:

$$
\begin{aligned}
\Rightarrow & \alpha_{1}=\left[\left(q_{\mathrm{fin}}-q_{0}\right)-\left(S_{0}(1)-S_{0}(0)\right)\right] \circ\left(S_{1}(1)-S_{1}(0)\right)^{\circ-1} \\
\Rightarrow & \bar{q}=q_{0}-\left[\left[\left(q_{\mathrm{fin}}-q_{0}\right)-\left(S_{0}(1)-S_{0}(0)\right)\right]\right. \\
& \left.\circ\left(S_{1}(1)-S_{1}(0)\right)^{\circ-1}\right] \circ S_{1}(0),
\end{aligned}
$$

where $q_{0}$ and $q_{\text {fin }}$ are the desired initial and final poses, respectively, $\alpha_{1}$ and $\bar{q}$ are the optimal parameters of a signal in the form given in Eq. 1, and ${ }^{0-1}$ is the Hadamart inverse as defined in [27]. Our solution proposes an implementation in which a combination of potential functions is used to describe the obstacles in the environment and the optimization problem is used to plan the motion while avoiding the obstacles. The cost function can be written as:

$$
\begin{array}{ll}
\min _{\bar{q}, \alpha_{1}, \ldots, \alpha_{M}} & \left\|\left[\begin{array}{c}
q(0)-q_{0} \\
q\left(t_{\mathrm{fin}}\right)-q_{\mathrm{fin}}
\end{array}\right]\right\|_{2}^{2}+\rho P\left(q, P_{O}\right) \\
\text { subject to } & q(t)=\bar{q}+S_{0}\left(\frac{t}{t_{\mathrm{fin}}}\right)+\sum_{i=1}^{M} \alpha_{i} \circ S_{i}\left(\frac{t}{t_{\mathrm{fin}}}\right),
\end{array}
$$

in which the first contribution guarantees that the desired initial and final poses are achieved, as for the free motion case reported in Eq. 3. The second term takes into account the distance, weighted by a suitable scaling scalar coefficient $\rho$, with respect to (w.r.t.) the obstacles.

Without any loss of generality, we assume here $N_{\mathrm{O}}$ spherical obstacles. Let assume $P_{\mathrm{O}}=\left\{P_{\mathrm{O}_{1}}, \ldots, P_{\mathrm{O}_{N_{\mathrm{O}}}}\right\}$ being the set containing the Cartesian coordinates of all the centers of the obstacles: we can hence define $P\left(q, P_{O}\right)$ as a potentialbased function [28] that sums up, for all the obstacles, a term inversely proportional to the minimum distance between the center of the obstacle and the closest generated joint trajectory, i.e.

$$
P\left(q, P_{O}\right)=\sum_{i=1}^{N_{O}} \frac{1}{m_{i}\left(q([0,1]), P_{\mathrm{O}_{i}}\right)^{2}}
$$

where $m_{i}$ is the minimum distance between the links of the robotic architecture and the $i-t h$ obstacle, defined as $m_{i}\left(q([0,1]), P_{\mathrm{O}_{i}}\right)=\min _{k}\left\{d\left(h_{k}(q([0,1])), P_{\mathrm{O}_{i}}\right)\right\} \cdot q([0,1])$ represents the trajectory of the joints from time zero to time 1 (recall that in Eq. 1 the whole trajectory depends on the variable $t / t_{\text {fin }}$ ). The distance between the $k-t h$ point of the rigid link of the manipulator defined by the forward kinematics function $h_{k}$, and the $i-t h$ sphere is

$d\left(h_{k}(q([0,1])), P_{\mathrm{O}_{i}}\right)=\max \left\{\min _{x \in h_{k}(q([0,1]))}\left(\left\|P_{\mathrm{O}_{i}}-x\right\|_{2}, R_{\mathrm{O}_{i}}\right)\right\}$,

with $R_{O_{i}}$ being the radius of the obstacle sphere. The optimization procedure incrementally enrolls functional modes until the trajectory is planned with a satisfactory residual error.
This choice is driven by the fact that a higher cardinality of the enrolled basis elements improves the accuracy of the reconstructed motion but also produces larger computational cost and increases the overall complexity of the problem (leading to a higher number of local minima [22], [29]). The general implementation of this algorithm is reported in Alg. 1.

\section{MAPPING BETWEEN KINEMATICS VIA IMPEDANCE CONTROL}

The framework introduced in the previous section enables the efficient and effective generation of human-inspired movements. However, the generated trajectory is defined in the joint space and, thus, is strictly related to the specific manipulator kinematic definition. In other terms, it can work only for robots with the same kinematic architecture as the one used for describing human motions. In the following, we will refer to this architecture as to the paradigmatic human arm. To overcome this limitation and generalize to any kinematic chain the approach reported in Alg. 1, we need to solve a mapping problem. This has been usually achieved in literature via optimization, minimizing the distance between the robot and the paradigmatic human arm: i.e. given the definition of an end effector (EE), a shoulder and an elbow - which can be easily identified in an anthropomorphic architecture [23], the goal is to minimize a cost function proportional to the sum of the area of the triangles between these points (for additional details the interested reader may refer to [23]). Another approach, namely Virtual Object Method introduced in [24], maps postures of hands with dissimilar kinematics, considering reference points on the two kinematic structures, and then mapping the movement of these reference points in the velocity domain. However, to the best of our knowledge, all the methods presented so far rely on time consuming optimization procedures, which need to be executed offline. To overcome this issue, we decided to map the movements using a different control-based approach. Since the mapping must preserve the main HL characteristics of the original planned movement, i.e. bell shaped velocity profiles, low jerk values etc, the control law needs to be transparent w.r.t. the motion dynamic characteristics. To this aim, we decided to implement a Cartesian Impedance Control (IC) [31]. More specifically, given the trajectory of the planned motion as it

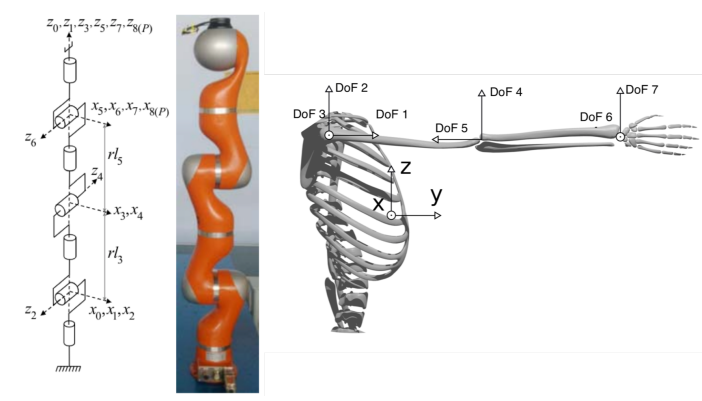

Fig. 2. Right plot. Kinematic model of the paradigmatic human arm. Left plot. Kinematic model of the Kuka LWR antrophomorphic manipulator. Figure partially adapted from [30] and [22] 
results from Alg. 1 and computed via forward-kinematics at the end-effector level, the following control law is adopted:

$$
\tau=M(q) \ddot{q}+c(q, \dot{q})+J_{1}(q)^{T}\left(K_{1} e_{1}+D_{1} \dot{e}_{1}\right),
$$

where $\tau$ represents the joint torques, $M(q)$ is the inertia matrix of the manipulator, $c(q, \dot{q})$ takes into account the Coriolis term and the gravity of the manipulator, $e_{1}=x^{o}-x$ is the Cartesian pose error at the EE, $\dot{e}_{1}$ is the error time derivative, and $J_{1}(q)$ is the Jacobian for the EE task. $K_{1}$ and $D_{1}$ are positive definite gain matrices.

In addition, since the majority of the anthropomorphic manipulators is redundant, i.e. the number of controllable joints is higher than the dimensionality of the end effector space, an additional Cartesian IC at the elbow level is introduced.

Implementing a control at the elbow is important not only to solve the redundacy problem [32], [33], [34] but also for increasing motion functional anthropomorphis. Indeed, it was proven that making the pose of the elbow of the slave robot as close as possible to the pose of the paradigmatic human arm enhances the HL of the commanded trajectories. Another aspect that increases functional anthropomorphism is the alignment of the end-effector of the robot with the paradigmatic human arm EE [23]. Note that, since the references of the end-effector and the elbow controllers are different, this may result in steady state error at the EE w.r.t. the reference trajectory. To have an exact mapping, i.e. zero tracking error, a null-space projection of torques produced by the second impedance controller is hence needed [32], [33], [34]. Our implementation of the second Cartesian IC can be achieved trough the following control law:

$$
v=J_{2}(q)^{T}\left(K_{2} e_{2}+D_{2} \dot{e}_{2}\right),
$$

where $J_{2}(q)$ is the elbow Jacobian, $e_{2}$ is the Cartesian position error (no orientation is considered) for the elbow, $\dot{e}_{2}$ its time derivative and $K_{2}, D_{2}$ positive definite gain matrices. This control action projects in the null space of the robotic manipulator EE. This is achieved with the projector $P_{1}(q)=$ $I-J_{1}(q)^{T} J_{1}(q)$.

The total control strategy can be hence written as:

$$
\tau=M(q)+c(q, \dot{q})+J_{1}(q)^{T}\left(K_{1} e_{1}+D_{1} \dot{e}_{1}\right)+P_{1}(q) J_{2}(q)^{T} v .
$$

\section{EXPERIMENTS}

To prove the effectiveness of our planning and mapping framework, we carried out a set of experiments on a real platform, i.e. a 7 Degrees of Freedom (DoFs) Kuka LWR+. The main difference between the paradigmatic human arm (see [21]) and the Kuka kinematics is that, in the latter case, the joint defining the shoulder, elbow and wrist degrees of freedom are not coincident. Note that our implementation can be applied to any robotic manipulator with redundant kinematic architecture (see Fig. 2).

Taking inspiration from [22], we identified four different sample movements that span the upper limb motion workspace, referred a first person view: T.1) is a translation from right to left side of the body in the transverse plane, a
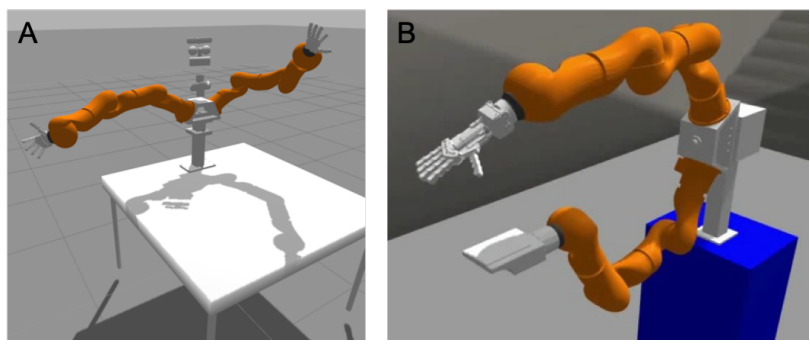

Fig. 3. Two different Kuka configurations. Figure A, robot in anthropomorphic dual arm configuration. Figure B, robot in industrial configuration, typically used to pick and maneuver objects from pallets. Note that in both cases only one arm (the right arm) is used.

typical movement used to manipulate objects on a table; T.2) is a movement towards a pose in front of the robot, in the sagittal plane; T.3) is an arm elevation mimicking a human motion towards a point upon the head; T.4) is a reaching movement reproducing a human motion towards the face, e.g. for self-feeding tasks. Numerical values of the initial and final joint configurations on the paradigmatic human arm kinematics are listed in Tab. []

Each task was planned in different conditions, i.e. with and without the presence of obstacles along the trajectory. The trajectories are then mapped on the Kuka kinematics in two different configurations: i) anthropomorphic configuration; ii) industrial configuration (see Fig. 3). Indeed, also in the latter case it is important to ensure HL to favour robot action predictability by the human operator [35]. Reference endeffector position defined on the human kinematics is scaled by a factor $L_{r} / L_{h}$, where $L_{r}$ and $L_{h}$ are the robot and human arm total arm length, respectively, when all the joints are set to zero, i.e. total arm extension. In Fig. 4 we show the end-effector commanded and real robotic EE pose in two sample cases. More specifically, we report the motion in the transverse plane (T.1) with two obstacles on the table $(20 \times 30 \mathrm{~cm}$ cylinder - height equal to 20 ; and $20 \times 30$ $\times 40 \mathrm{~cm}$ paralleliped - height equal to 40 , at a center-tocenter distance of approx. $10 \mathrm{~cm}$ ), and one movement in the medial plane (T.4) for the free-movement case (see also Fig. 5). Results show a good approximation of the commanded end-effector motion, with a mean error between the reference and real EE position lower than $5 * 10^{-2} \mathrm{~m}$, and an EE average orientation error lower than $10^{-1}$. All the other tested movements presented similar performances, details are not reported here for sake of space. The corresponding

TABLE I

INITIAL $\left(q_{0}\right)$ AND FINAL $\left(q_{\text {fin }}\right)$ JOINT POSTURE FOR THE FOUR TASKS. ANGLES ARE EXPRESSED IN RADIANTS AND DEFINED FOR THE PARADIGMATIC HUMAN ARM KINEMATICS.

\begin{tabular}{rlrrrrrrr}
\hline \multicolumn{2}{c}{ Task } & dof 1 & dof 2 & dof 3 & dof 4 & dof 5 & dof 6 & dof 7 \\
\hline T.1 & $q_{0}$ & -0.31 & -0.58 & -0.8 & 0.47 & -1.23 & 0.22 & 0.65 \\
& $q_{\text {fin }}$ & -0.33 & 0.34 & -0.39 & 0.44 & -0.69 & 0.36 & 0.15 \\
\hline T.2 & $q_{0}$ & 0.09 & -0.79 & -0.62 & 0.65 & -1.50 & 0.06 & 0.76 \\
& $q_{\text {fin }}$ & 0.65 & -0.40 & 0.07 & 0.79 & -1.32 & 0.87 & 0.63 \\
\hline T.3 & $q_{0}$ & -0.48 & -0.07 & -0.71 & 0.03 & -0.86 & 0.52 & 0.47 \\
& $q_{\text {fin }}$ & -1.67 & -0.68 & -0.33 & -0.21 & -0.09 & -0.26 & 0.52 \\
\hline T.4 & $q_{0}$ & -0.28 & -0.86 & -0.92 & 0.86 & -1.52 & -0.12 & 0.77 \\
& $q_{\text {fin }}$ & 0.65 & -0.78 & -0.12 & 2.18 & -1.52 & 0.57 & 0.59 \\
\hline
\end{tabular}



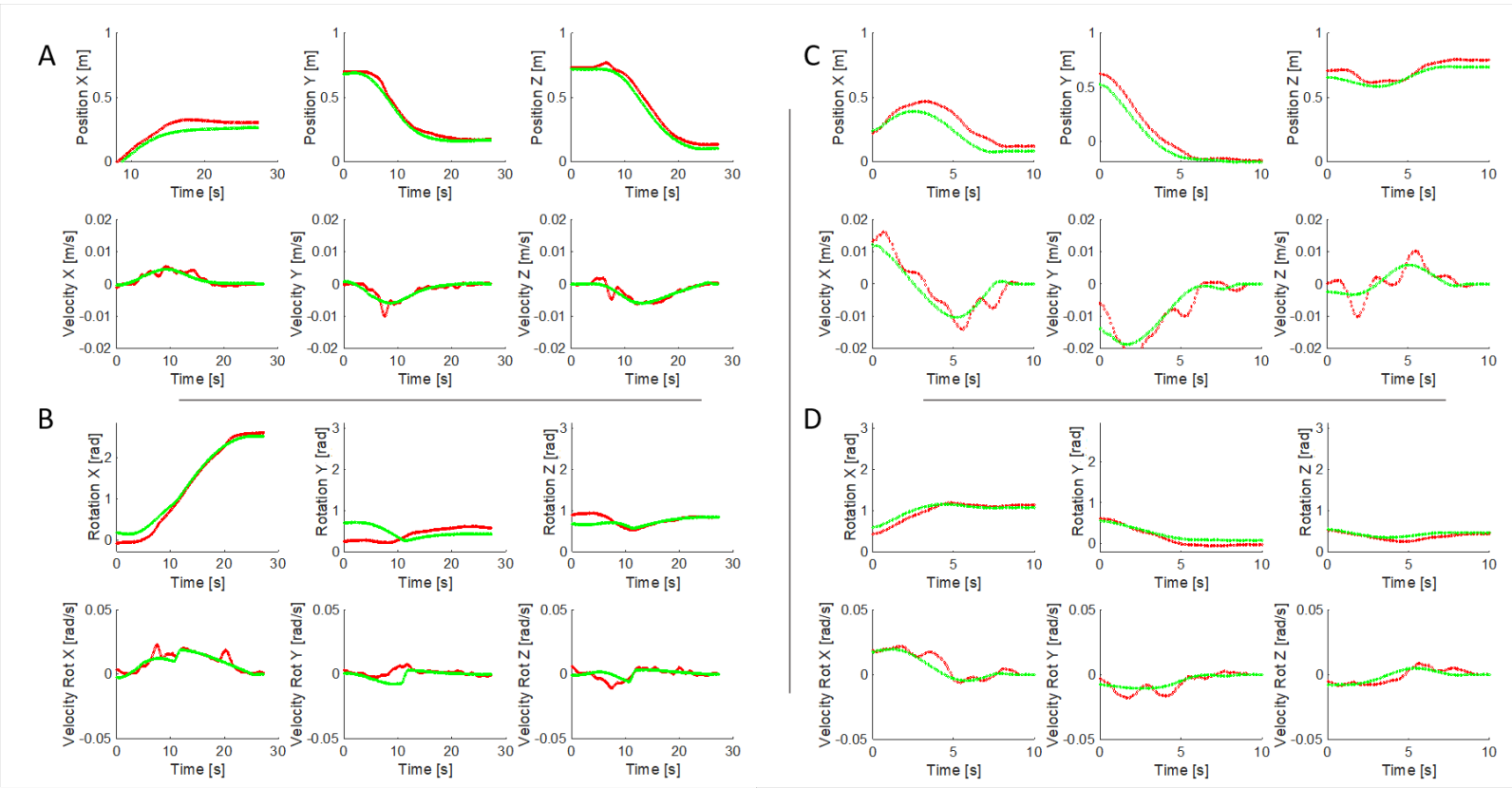

Fig. 4. Mapped motions. Subplot A Position (first row) and velocity (second row) of the reference (green line) and the real (red line) end-effector position for task T.1 with two obstacles. Subplot B Position (first row) and velocity (second row) of the reference (green line) and the real (red line) end-effector orientation for task T.1 with two obstacles. Subplot C Position (first row) and velocity (second row) of the reference (green line) and the real (red line) end-effector position for task T.4 with no obstacle. Subplot D Position (first row) and velocity (second row) of the reference (green line) and the real (red line) end-effector otientation for task T.4 with no obstacle.

velocities are depicted in Fig. 4 Notwithstanding the presence of oscillations due to vibrations of the manipulator not related to our control law, the robot movement shows bell-shaped velocity profiles with jerk values always lower than $10^{-2}$, in line with the observations in [36].

The overall movement is depicted in Fig. 5 for two sample actions (T.1 and T.4), i.e. for the free motion and the two-obstacle case. See also the attached video (https: //youtu.be/FHOsVVXBV74).

\section{Discussions AND CONCLUSIONS}

In this paper we propose a novel technical framework for human-like motion generation for anthropomorphic robots. The goal is achieved through two main building blocks. First, we discussed a motion generation algorithm based on the optimal combination of human data-driven functional modes [21]. This method was preliminarily theorized in [22] and is here extended for its usability with generic redundant anthropomorphic manipulators, whose kinematic architecture differs from the one used to extract human functional modes. This is achieved through a mapping algorithm, which exploits torque control of the manipulator. More specifically, we implemented a Cartesian impedance control at the end-effector level. Functional anthropomorphism of the overall motion is preserved via a second Cartesian impedance controller at the elbow level. This applies an additional torque in the nullspace of the end-effector Cartesian controller to keep the robot elbow as close as possible to the reference of the human elbow. The reference pose commanded is the movement planned on the human kinematics, scaled by a proportionality factor. Our experiments show that such control strategy is able to on-line map the desired trajectory of the paradigmatic human arm at the robot end-effector level. Robot trajectories appear to maintain the basic characteristics of human-likeliness of the commanded poses, i.e. low jerk values, bell-shaped velocity profile. Note that - due to kinematic constraints - the second controller is not meant to achieve zero error, while the endeffector is expected to follow with a good approximation the desired trajectory (position and orientation).

In the future developments, the approach described in this paper will be tested with other manipulators and configurations and will be integrated in a more general framework together with a vision based target and obstacle identifier. It is important to underline here that the aim of this work is not to solve planning problems but instead to propose a new method for intrinsically embedding human-likeness in motion generation. Future work will encompass the evaluation with sophisticated planning problems and state of the art planning benchmarks. The outcomes of this paper could successfully inform the next developments for the control of redundant anthropomorphic co-bots in industrial settings and for human assistance. Indeed, thank to the human-likeness of the robot trajectories, the robot interaction with the humans in collaborative tasks, e.g. for synergistic action completion, or during robot-assisted actions, e.g. self feeding, will be performed in a predictable, ergonomic and safe manner. This will finally and likely increase the acceptability of robotic technology by the human user. Under this regard, the analysis of the ergonomy of humans interacting with robotic manipulators implementing the control strategies reported in this paper (see e.g. [37] ), and the evaluation of the perceived human-likeliness via experiments with human participants are already envisioned. 

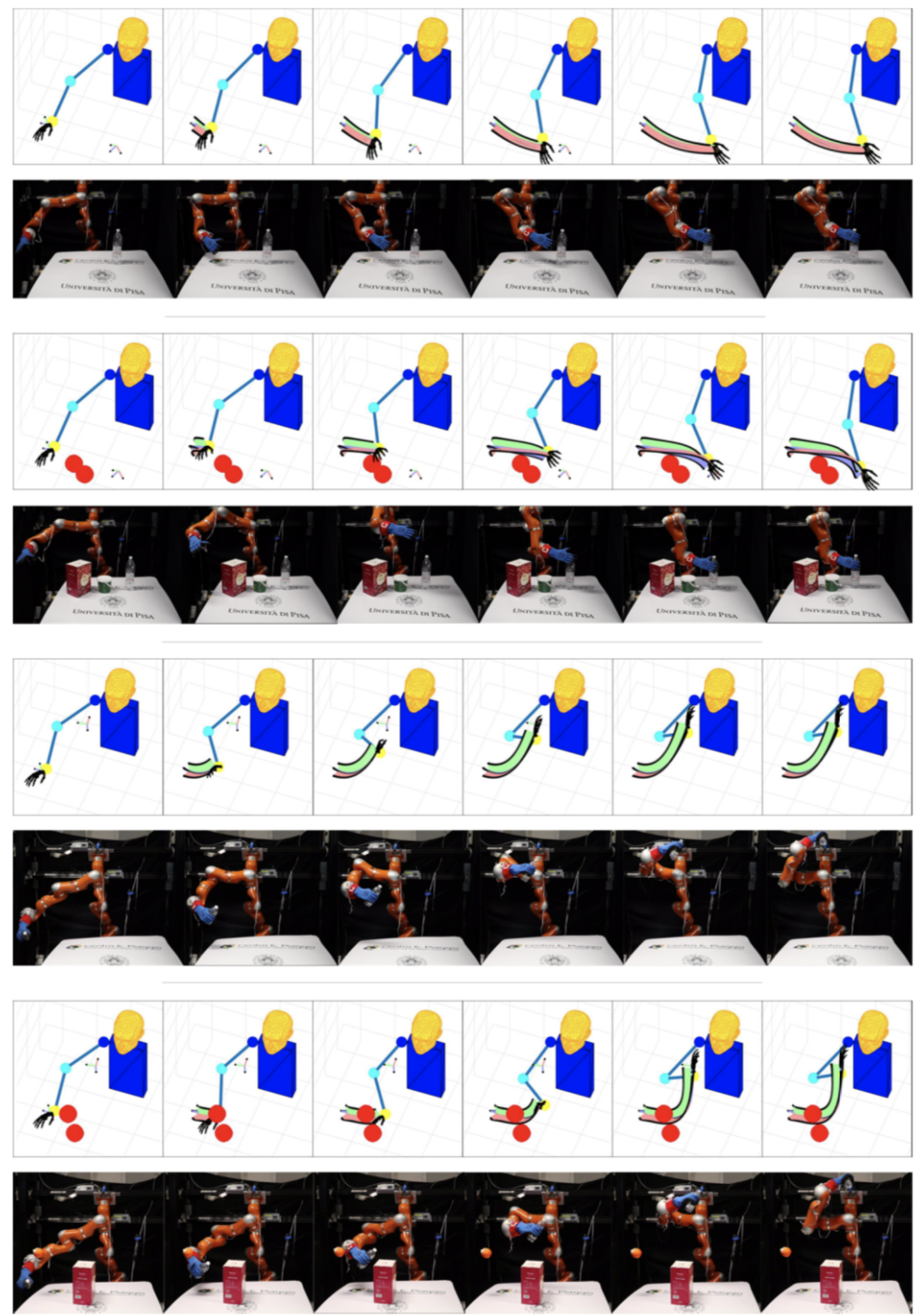

Fig. 5. From top to bottom, snapshots of human reference and real robot execution of T.1 free motion, T.1 with two obstacles; T.4 free motion; T.4 with two obstacles. First controller param: $K_{x}=100 ; K_{y}=100 ; K_{z}=100 ; K_{r x}=1.5 ; K_{r y}=1.5 ; K_{r z}=1.5 ; D_{x}=2.0 ; D_{y}=2.0 ; D_{z}=2.0 ; D_{r x}=1.0 ; D_{r y}=1.0$; $D_{r z}=1.0$. Second controller param: $K_{x}=5.0 ; K_{y}=5.0 ; K_{z}=5.0 ; K_{r x}=0 ; K_{r y}=0 ; K_{r z}=0 ; D_{x}=0 ; D_{y}=0 ; D_{z}=0 ; D_{r x}=0 ; D_{r y}=0 ; D_{r z}=0$. 


\section{REFERENCES}

[1] S. Wischniewski and A. Ajoudani, "How can assistive robotics improve personal and work life? a million dollar question," IEEE Robotics Automation Magazine, vol. 26, no. 2, pp. 106-106, June 2019.

[2] C. Bartneck, D. Kulić, E. Croft, and S. Zoghbi, "Measurement instruments for the anthropomorphism, animacy, likeability, perceived intelligence, and perceived safety of robots," International journal of social robotics, vol. 1, no. 1, pp. 71-81, 2009.

[3] J. Fink, "Anthropomorphism and human likeness in the design of robots and human-robot interaction," in International Conference on Social Robotics. Springer, 2012, pp. 199-208.

[4] L. D. Riek, T.-C. Rabinowitch, B. Chakrabarti, and P. Robinson, "How anthropomorphism affects empathy toward robots," in Proceedings of the 4th ACM/IEEE international conference on Human robot interaction. ACM, 2009, pp. 245-246.

[5] A. Edsinger and C. C. Kemp, "Two arms are better than one: A behavior based control system for assistive bimanual manipulation," in Recent progress in robotics: Viable robotic service to human. Springer, 2007, pp. 345-355.

[6] T. Mukai, S. Hirano, H. Nakashima, Y. Sakaida, and S. Guo, "Realization and safety measures of patient transfer by nursing-care assistant robot riba with tactile sensors," Journal of Robotics and Mechatronics, vol. 23, no. 3, pp. 360-369, 2011.

[7] B. R. Duffy, "Anthropomorphism and the social robot," Robotics and autonomous systems, vol. 42, no. 3, pp. 177-190, 2003.

[8] J. von Zitzewitz, P. M. Boesch, P. Wolf, and R. Riener, "Quantifying the human likeness of a humanoid robot," International Journal of Social Robotics, vol. 5, no. 2, pp. 263-276, 2013.

[9] P. Morasso, "Spatial control of arm movements," Experimental brain research, vol. 42, no. 2, pp. 223-227, 1981.

[10] T. Flash and N. Hogan, "The coordination of arm movements: an experimentally confirmed mathematical model," The journal of Neuroscience, vol. 5, no. 7, pp. 1688-1703, 1985.

[11] A. Piazzi and A. Visioli, "Global minimum-jerk trajectory planning of robot manipulators," IEEE transactions on industrial electronics, vol. 47, no. 1, pp. 140-149, 2000.

[12] M. D. K. Breteler, S. C. Gielen, and R. G. Meulenbroek, "End-point constraints in aiming movements: effects of approach angle and speed," Biological Cybernetics, vol. 85, no. 1, pp. 65-75, 2001.

[13] C. A. Avizzano, "Guided latent space regression for human motion generation," Robotics and Autonomous Systems, vol. 61, no. 4, pp. 340-350, 2013.

[14] Z. Zhang, Z. Li, Y. Zhang, Y. Luo, and Y. Li, "Neural-dynamic-methodbased dual-arm cmg scheme with time-varying constraints applied to humanoid robots." IEEE Trans. Neural Netw. Learning Syst., vol. 26, no. 12, pp. 3251-3262, 2015.

[15] I. Mordatch, K. Lowrey, G. Andrew, Z. Popovic, and E. V. Todorov, "Interactive control of diverse complex characters with neural networks," in Advances in Neural Information Processing Systems, 2015, pp. 3132 3140 .

[16] X. B. Peng, G. Berseth, and M. Van de Panne, "Terrain-adaptive locomotion skills using deep reinforcement learning," ACM Transactions on Graphics (TOG), vol. 35, no. 4, p. 81, 2016.

[17] M. V. Liarokapis, P. K. Artemiadis, P. T. Katsiaris, K. J. Kyriakopoulos, and E. S. Manolakos, "Learning human reach-to-grasp strategies: Towards emg-based control of robotic arm-hand systems," in 2012 IEEE International Conference on Robotics and Automation, May 2012, pp. 2287-2292.

[18] N. B. Figueroa Fernandez and A. Billard, "Modeling compositions of impedance-based primitives via dynamical systems." in In Proceedings of the Workshop on Cognitive Whole-Body Control for Compliant Robot Manipulation (COWB-COMP), no. CONF, 2018.

[19] I. Mordatch, J. M. Wang, E. Todorov, and V. Koltun, "Animating human lower limbs using contact-invariant optimization," ACM Transactions on Graphics (TOG), vol. 32, no. 6, p. 203, 2013.

[20] S. Miossec and A. Kheddar, "Human motion in cooperative tasks: Moving object case study," in Robotics and Biomimetics, 2008. ROBIO 2008. IEEE International Conference on. IEEE, 2009, pp. 1509-1514.

[21] G. Averta, C. Della Santina, E. Battaglia, F. Felici, M. Bianchi, and A. Bicchi, "Unvealing the principal modes of human upper limb movements through functional analysis," Frontiers in Robotics and AI, vol. 4, p. 37, 2017.

[22] G. Averta, C. Della Santina, G. Valenza, A. Bicchi, and M. Bianchi, "Exploiting upper-limb functional principal components for human-like motion generation of anthropomorphic robots," Journal of NeuroEngineering and Rehabilitation.

[23] M. Liarokapis, C. P. Bechlioulis, P. K. Artemiadis, and K. J. Kyriakopoulos, "Deriving humanlike arm hand system poses," Journal of Mechanisms and Robotics, vol. 9, no. 1, p. 011012, 2017.
[24] G. Gioioso, G. Salvietti, M. Malvezzi, and D. Prattichizzo, "Mapping synergies from human to robotic hands with dissimilar kinematics: an approach in the object domain," IEEE Transactions on Robotics, vol. 29, no. 4, pp. 825-837, 2013.

[25] A. M. Zanchettin, L. Bascetta, and P. Rocco, "Achieving humanlike motion: Resolving redundancy for anthropomorphic industrial manipulators," IEEE Robotics \& Automation Magazine, vol. 20, no. 4, pp. 131-138, 2013.

[26] M. V. Liarokapis, P. K. Artemiadis, and K. J. Kyriakopoulos, "Functional anthropomorphism for human to robot motion mapping," in 2012 IEEE RO-MAN: The 21st IEEE International Symposium on Robot and Human Interactive Communication. IEEE, 2012, pp. 31-36.

[27] R. Reams, "Hadamard inverses, square roots and products of almost semidefinite matrices," Linear Algebra and its Applications, vol. 288, pp. 35-43, 1999.

[28] Y. K. Hwang and N. Ahuja, "A potential field approach to path planning," IEEE Transactions on Robotics and Automation, vol. 8, no. 1, pp. 2332, 1992.

[29] G. Averta, F. Angelini, M. Bonilla, M. Bianchi, and A. Bicchi, "Incrementality and hierarchies in the enrollment of multiple synergies for grasp planning," IEEE Robotics and Automation Letters, vol. 3, no. 3, pp. 2686-2693, 2018.

[30] A. Jubien, M. Gautier, and A. Janot, "Dynamic identification of the kuka lwr robot using motor torques and joint torque sensors data," IFAC Proceedings Volumes, vol. 47, no. 3, pp. 8391-8396, 2014.

[31] N. Hogan, "Impedance control-an approach to manipulation. i-theory. ii-implementation. iii-applications," ASME Transactions Journal of Dynamic Systems and Measurement Control B, vol. 107, pp. 1-24, 1985.

[32] O. Khatib, "A unified approach for motion and force control of robot manipulators: The operational space formulation," IEEE Journal on Robotics and Automation, vol. 3, no. 1, pp. 43-53, 1987.

[33] - "Inertial properties in robotic manipulation: An object-level framework," The international journal of robotics research, vol. 14, no. 1, pp. 19-36, 1995.

[34] A. Dietrich, C. Ott, and A. Albu-Schäffer, "An overview of null space projections for redundant, torque-controlled robots," The International Journal of Robotics Research, vol. 34, no. 11, pp. 1385-1400, 2015.

[35] A. M. Zanchettin, L. Bascetta, and P. Rocco, "Acceptability of robotic manipulators in shared working environments through human-like redundancy resolution," Applied ergonomics, vol. 44, no. 6, pp. 982989, 2013.

[36] T. Flash, "The control of hand equilibrium trajectories in multi-joint arm movements," Biological cybernetics, vol. 57, no. 4-5, pp. 257-274, 1987.

[37] L. Peternel, C. Fang, N. Tsagarakis, and A. Ajoudani, "A selective muscle fatigue management approach to ergonomic human-robot co-manipulation," Robotics and Computer-Integrated Manufacturing, vol. 58, pp. 69 - 79, 2019. [Online]. Available: http://www.sciencedirect.com/science/article/pii/S0736584518303132 